\title{
Enhanced Biotransformation Productivity of Gamma-Decalactone from Ricinoleic Acid Based on the Expanded Vermiculite Delivery System
}

\author{
Shimin Guan, Shaofeng Rong, Mengze Wang, Baoguo Cai, Qianqian Li, and Shuo Zhang* \\ Department of Biological Engineering, Shanghai Institute of Technology, Shanghai 201418, P.R. China
}

\author{
Received: April 29, 2019 \\ Revised: July 1, 2019 \\ Accepted: July 2, 2019 \\ First published online \\ July 10, 2019 \\ *Corresponding author \\ Phone: +86-021-60877260; \\ E-mail: zhangshuo0430@163.com \\ pISSN 1017-7825, eISSN 1738-8872 \\ Copyright(C) 2019 by \\ The Korean Society for Microbiology \\ and Biotechnology
}

\begin{abstract}
Natural gamma-decalactone (GDL) produced by biotransformation is an essential food additive with a peach-like aroma. However, the difficulty of effectively controlling the concentration of the substrate ricinoleic acid (RA) in water limits the biotransformation productivity, which is a bottleneck for industrialization. In this study, expanded vermiculite $(\mathrm{E}-\mathrm{V})$ was utilized as a carrier of RA to increase its distribution in the medium. E-V and three commonly used organic compounds were compared with respect to their effects on the biotransformation process, and the mechanism was revealed. Scanning electron microscopy, Fourier transform infrared spectroscopy and thermogravimetric analysis indicated that RA was physically adsorbed onto the surface of and inside E-V instead of undergoing a chemical reaction, which increased the opportunity for interactions between microorganisms and the substrate. The highest concentration of GDL obtained in the medium with E-V was $6.2 \mathrm{~g} / 1$, which was $50 \%$ higher than that in the reference sample. In addition, the presence of E-V had no negative effect on the viability of the microorganisms. This study provides a new method for producing natural GDL through biotransformation on an industrial scale.
\end{abstract}

Keywords: Biotransformation, gamma-decalactone, ricinoleic acid, expanded vermiculite

\section{Introduction}

Lactones are molecules resulting from hydroxy acid cyclization, which comprises a carbon cycle with one oxygen atom. Lactones are attractive flavor additives for food and pharmaceutical products due to their fruit aroma [1]. Among these compounds, gamma-decalactone (GDL) is the most important, with a characteristic peach-like flavor detectable at low concentrations below $5 \mathrm{mg} / 1$ [2]. Traditionally, GDL is obtained by direct extraction from fruits and plants or by chemical synthesis. However, the aromatic quality of synthetic GDL is lower than that of natural GDL. The yield from the extraction method is limited and influenced by geographical and climatic conditions, resulting in a high price (US_\$3,000/ kg). In recent years, the utilization of microorganisms and enzymes for the production of flavor compounds has received a great deal of attention. The main driving force is that the flavor compounds produced by this biotechnological method can be labelled "natural" with much lower cost [2].
In the biotransformation process, several microorganisms have been selected for their potential to produce aroma substances, including Pseudomonas, Sporobolomyces, Pichia, Candida, Rhodotorula, and Yarrowia lipolytica [2]. Among these microorganisms, Y. lipolytica is reported to have the strongest productivity [3]. Castor oil is commonly utilized as a substrate for the production of GDL by microorganisms [3-5]. However, the castor oil should be hydrolyzed to ricinoleic acid (RA), which accounts for $86 \%$ of castor oil as the main component, prior to entering the bioconversion cycle [6]. Inside the cells of microorganisms, RA enters the mitochondria. After four $\beta$-oxidation cycles, RA degrades to 4-hydroxy-decanoyl-CoA, which then cyclizes to GDL [3]. Moradi et al. reported that the highest concentration of GDL was 62.4 and $52.9 \mathrm{mg} / 1$ from $1.5 \%$ RA and $2.5 \%$ castor oil, respectively. [7] Rong et al. proved that L-carnitine shortened the biotransformation period by approximately $10 \mathrm{~h}$ and increased GDL production by $19.5 \%$ when RA was utilized by Saccharomyces cerevisiae MF013 [8].

However, the low solubility of RA in water limits the 
probability that microorganisms will come into contact with the substrate, which results in a low yield of GDL. To increase the solubility of the substrate, many surfactants have been added to the media. Nelma Gomes reported much more accumulation of GDL, approximately $1.8 \mathrm{~g} / \mathrm{l}$, after the addition of Tween 80 [9]. Aguedo discovered that two tested ionic surfactants could increase the solubility and transport rate of RA [10, 11]. However, removing surfactants from the final products in the extraction phase is difficult. In addition, some surfactants have been reported to change the permeability of the cell membrane during biotransformation, which may exert a toxic effect on the cell membrane at certain concentrations [12]. Therefore, seeking a new approach to increase the dispersion of the substrate without inhibiting cellular activity is essential to improve the production of GDL.

Adsorption is recognized as a promising technique due to its ease of operation, easy availability, high efficiency and relative low cost of application in the biotransformation process. Expanded vermiculite $(\mathrm{E}-\mathrm{V})$ is a natural, multilayerstructure magnesium aluminium silicate inorganic mineral with many crystal interpacket spaces acting as pores; the volume of the material can be expanded by 8 to 30 times depending on the texture and the thermal conditions used [13]. Charges located between the sheets contribute to the good ion-exchange properties of E-V. Owing to its remarkable abundance and cheap price, E-V is widely applied in the agricultural, industrial and environmental fields as a micro-porous, non-toxic, absorbent and sustainedrelease material $[14,15]$. Because $\mathrm{E}-\mathrm{V}$ is an inert inorganic material, toxic effects on cells and the phenomenon of emulsification can be avoided.

In this study, we utilized E-V as a carrier of RA to increase the distribution and controlled release of the substrate in media and compared its effect on biotransformation with three commonly utilized hydrophilic organic solvents: dimethylsulfoxide (DMSO), ethylene glycol and acetone [16]. Changes in cell proliferation, $\mathrm{pH}$, and the concentrations of RA and GDL were monitored every $12 \mathrm{~h}$. Scanning electron microscopy (SEM), gas chromatography (GC) and high-performance liquid chromatography (HPLC) were utilized to reveal the adsorption mechanism of the EV-RA complexes and the relationship between the RA release rate and GDL productivity. To the best of our knowledge, most studies have focused on increasing GDL productivity using surfactants or immobilization of microorganisms; only a few studies have focused on the utilization of an RA-controlled delivery system to disperse the substrate. Our research is the first attempt to use E-V with the purpose of efficiently producing GDL at the industrial level.

\section{Materials and Methods}

\section{Microorganisms and Reagents}

Y. lipolytica (CGMCC 2.2087), used for GDL biotransformation, was obtained from the China General Microbiological Culture Collection Center (China). The RA substrate was obtained from Zibo Zhoucun Mingdong Chem, Ltd. (China). E-V was obtained from Suzhou Yilufa Environmental Protection Technology, Ltd. (China). The GDL standard was provided by Adamas (China). Analytical-grade ethanol, DMSO, ethylene glycol, acetone and other chemicals were purchased from Sinopharm Chemical Reagent, Ltd. (China).

\section{Cultivation of Microorganisms}

Y. lipolytica (CGMCC 2.2087) was cultured for $30 \mathrm{~h}$ on agar slant culture medium (20 g/l agar, $10 \mathrm{~g} / 1$ glucose, $10 \mathrm{~g} / 1$ peptone, and $12 \mathrm{~g} / 1$ yeast extract) at $28^{\circ} \mathrm{C}$ and inoculated in a $250 \mathrm{ml}$ baffled Erlenmeyer flask containing $50 \mathrm{ml}$ of YPD medium $(10 \mathrm{~g} / 1$ glucose, $10 \mathrm{~g} / 1$ peptone, and $12 \mathrm{~g} / 1$ yeast extract). Flasks were shaken at $200 \mathrm{rpm}$ and $28^{\circ} \mathrm{C}$ for $24 \mathrm{~h}$ until the cultures reached the late-logarithmic growth phase. These suspensions were used to inoculate the biotransformation media.

\section{Preparation of RA and E-V Complexes}

RA was embedded in E-V through the following steps. A 1:1 mass ratio of RA to $\mathrm{E}-\mathrm{V}$ was stirred for $30 \mathrm{~min}$ in a $50^{\circ} \mathrm{C}$ water bath. The embedded complexes were added to the biotransformation medium and were subjected to wet sterilization at $121^{\circ} \mathrm{C}$ for $15 \mathrm{~min}$. The adsorption capacity of $\mathrm{E}-\mathrm{V}$ was estimated as follows:

\author{
Adsorption capacity $(\mathrm{g} / \mathrm{g})=$ \\ Weight of embedded material (g)-Weight of E-V (g) \\ Weight of $\mathrm{E}-\mathrm{V}(\mathrm{g})$
}

\section{Bioconversion of RA to GDL}

Two different strategies of GDL production were compared: the addition of E-V and organic solvents. For the medium with E-V, $15 \%$ Y. lipolytica cell suspensions were inoculated directly into $30 \mathrm{ml}$ biotransformation medium containing $80 \mathrm{~g} / 1 \mathrm{E}-\mathrm{V}-\mathrm{RA}$ complex (containing $40 \mathrm{~g} / \mathrm{l} \mathrm{RA}$ ), $7 \mathrm{~g} / \mathrm{l} \mathrm{MgSO}_{4}, 7 \mathrm{~g} / 1 \mathrm{~K}_{2} \mathrm{HPO}_{4}, 15 \mathrm{~g} / \mathrm{l}$ glycerol, and $0.1 \mathrm{~g} / \mathrm{l}$ carnitine in ultrapure water at the natural $\mathrm{pH}$ of the medium. For the medium with organic solvents, after 1\% DMSO, ethylene glycol, and acetone were added individually to medium containing $40 \mathrm{~g} / 1 \mathrm{RA}, 7 \mathrm{~g} / 1 \mathrm{MgSO}_{4}, 7 \mathrm{~g} / 1 \mathrm{~K}_{2} \mathrm{HPO}_{4}, 15 \mathrm{~g} / \mathrm{l}$ glycerol, and $0.1 \mathrm{~g} / 1$ carnitine in a sterile environment, $15 \%$ cell suspensions were inoculated. The culture was agitated at $250 \mathrm{rpm}$ at $28^{\circ} \mathrm{C}$ for $84 \mathrm{~h}$. A medium without E-V or organic solvents was prepared as a reference. In all cases, samples were collected aseptically every $12 \mathrm{~h}$. 


\section{Analytical Methods}

a) Cell concentration

The free cell concentration was estimated by UV spectrophotometry. Each sample taken from the medium was diluted 15 times for triplicate measurements.

b) Characterization of embedded material Images of the E-V, embedded complex, and adhered $Y$. lipolytica were obtained through SEM (Hitachi S-3400N, Japan) at an accelerating voltage of $15.0 \mathrm{kV}$. Samples were fixed on a specimen holder with aluminium tape and sputtered with gold under high-vacuum conditions.

Thermogravimetric (TG) analysis (NETZSCH, Germany) of the E-V, RA, and embedded material was performed. Nitrogen was used as the shielding gas during the analysis at a flow rate of $20 \mathrm{ml} / \mathrm{min}$, and the temperature ranged from $30-700^{\circ} \mathrm{C}$ at a rate of $10^{\circ} \mathrm{C} / \mathrm{min}$.

Differential thermogravimetric (DTG) analysis was performed by calculating the first derivative of the TG curve or the rate of mass change rate with respect to temperature and time. The mathematical expression is:

$\operatorname{DTG}\left(\% /{ }^{\circ} \mathrm{C}\right)=\frac{\mathrm{d} W / \mathrm{d} t}{\mathrm{~d} T / \mathrm{d} t}$

where $W$ is the mass fraction, $T$ is the programmed temperature, and $t$ is time.

Fourier transform infrared (FTIR) spectroscopy (Thermo Fisher Scientific Inc., USA) was used to analyze the E-V, RA, and embedded material in the $4,000-400 \mathrm{~cm}^{-1}$ range. Samples were blended with $\mathrm{KBr}$ to form pellets.

c) $\mathrm{pH}$ Measurement

Samples were collected for $\mathrm{pH}$ analysis with a $\mathrm{pH}$ meter (PHS-3C, Leici, China) every $12 \mathrm{~h}$ in triplicate.

d) Analysis of components in fermentation broth

Samples were collected at intervals to determine the concentrations of GDL and RA. Pure chemical compounds $(\geq 98 \%)$ were used as standards. Each sample was mixed with certain volumes of ethanol, gently shaken for $5 \mathrm{~min}$, and then centrifuged at $4,500 \times g$. The supernatant was collected for testing. GDL was analyzed by using a GC instrument (Agilent 6890N, Agilent Technologies, Ltd., USA) equipped with a 19091J-433 HP-5 chromatographic column (30 m $\times$ $0.25 \mathrm{~mm} \times 0.25 \mu \mathrm{m})$. The split ratio was $30: 1$, and $\mathrm{N}_{2}$ was the carrier gas. The operating conditions for the analysis were as follows: a $0.2 \mu \mathrm{l}$ sample was injected at $250^{\circ} \mathrm{C}$; the detector temperature was $300^{\circ} \mathrm{C}$; and the oven temperature was maintained at $120^{\circ} \mathrm{C}$ for $2 \mathrm{~min}$, increased to $205^{\circ} \mathrm{C}$ at a rate of $30^{\circ} \mathrm{C} / \mathrm{min}$, raised to $215^{\circ} \mathrm{C}$ at a rate of $4^{\circ} \mathrm{C} / \mathrm{min}$, raised to $280^{\circ} \mathrm{C}$ at a rate of $20^{\circ} \mathrm{C} / \mathrm{min}$ and held constant for $3 \mathrm{~min}$.

The concentration of RA was monitored using an HPLC instrument (Agilent 1260, Agilent Technologies, Ltd., USA) equipped with an XDB-C18 column $(250 \mathrm{~mm} \times 4.5 \mathrm{~mm})$. Approximately $2 \mu \mathrm{l}$ sample volumes were injected into the column and eluted at a flow rate of $1 \mathrm{ml} / \mathrm{min}$ with $5 \% \mathrm{v} / \mathrm{v}$ of $0.1 \%$ $\mathrm{v} / \mathrm{v}$ phosphoric acid/water and $95 \% \mathrm{v} / \mathrm{v}$ acetonitrile, and a detection wavelength of $205 \mathrm{~nm}$ was used.

The conversion rate of the product was estimated as follows:

Conversion rate $(\%)=$

Concentration of GDL/MW of GDL

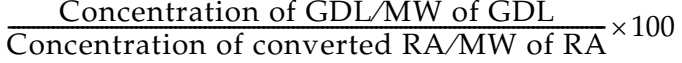

where MW is the molecular weight.

\section{Results}

\section{Effect of E-V and Organic Solvents on the Biotransformation Process}

The effects of E-V, DMSO, ethylene glycol and acetone on biotransformation were compared with the reference results. The cell viability, $\mathrm{pH}, \mathrm{RA}$ consumption and GDL yield were evaluated (Fig. 1). Regarding cell viability, there was a slight decrease in all the cultures with additions, except for the reference culture, in which the OD increased from 0.426 to 0.453 during biotransformation. However, the concentration of cells in the culture with ethylene glycol declined the most, from 0.426 to 0.328 (Fig. 1A). This indicates that ethylene glycol may have a stronger toxic effect on the proliferation of microorganisms. In terms of $\mathrm{pH}$, there was not much variation during biotransformation, except in the medium with ethylene glycol, in which the $\mathrm{pH}$ decreased from 6.36 to 4.59 (Fig. 1B).

The highest yield of GDL was $6.2 \mathrm{~g} / 1$ in the culture with $\mathrm{E}-\mathrm{V}$ at $60 \mathrm{~h}$, which was 50\% higher than that in the control (Fig. 1C). The second highest yield was obtained in the culture with acetone, which was $5.32 \mathrm{~g} / 1$. The yields in the medium with DMSO and the reference were almost the same, with values of $4.34 \mathrm{~g} / 1$ and $4.14 \mathrm{~g} / \mathrm{l}$, respectively. The lowest yield was obtained from the medium with ethylene glycol, which was $1.125 \mathrm{~g} / \mathrm{l}$. Obviously, the effect of ethylene glycol on biotransformation is negative. From $48 \mathrm{~h}$ to $60 \mathrm{~h}$, a high production rate of GDL was obtained in all the cultures, and this rate gradually slowed after $60 \mathrm{~h}$. Among these media, the highest production rate was achieved in the culture with E-V, which was $0.517 \mathrm{~g} / \bullet \bullet h$. (Table 2).

Fig. 1D indicates that the highest consumption of RA was achieved in the medium with E-V, which was $14.24 \mathrm{~g} / 1$. The reason for this phenomenon was speculated to be that the sustained release effect of E-V increased the utilization of RA. The second highest consumption of RA was obtained in the medium with acetone, which was $13.15 \mathrm{~g} / 1$. The lowest consumption of RA was obtained in the medium with ethylene glycol, which was $5.92 \mathrm{~g} / 1$. The concentrations of RA in the medium with DMSO and the 

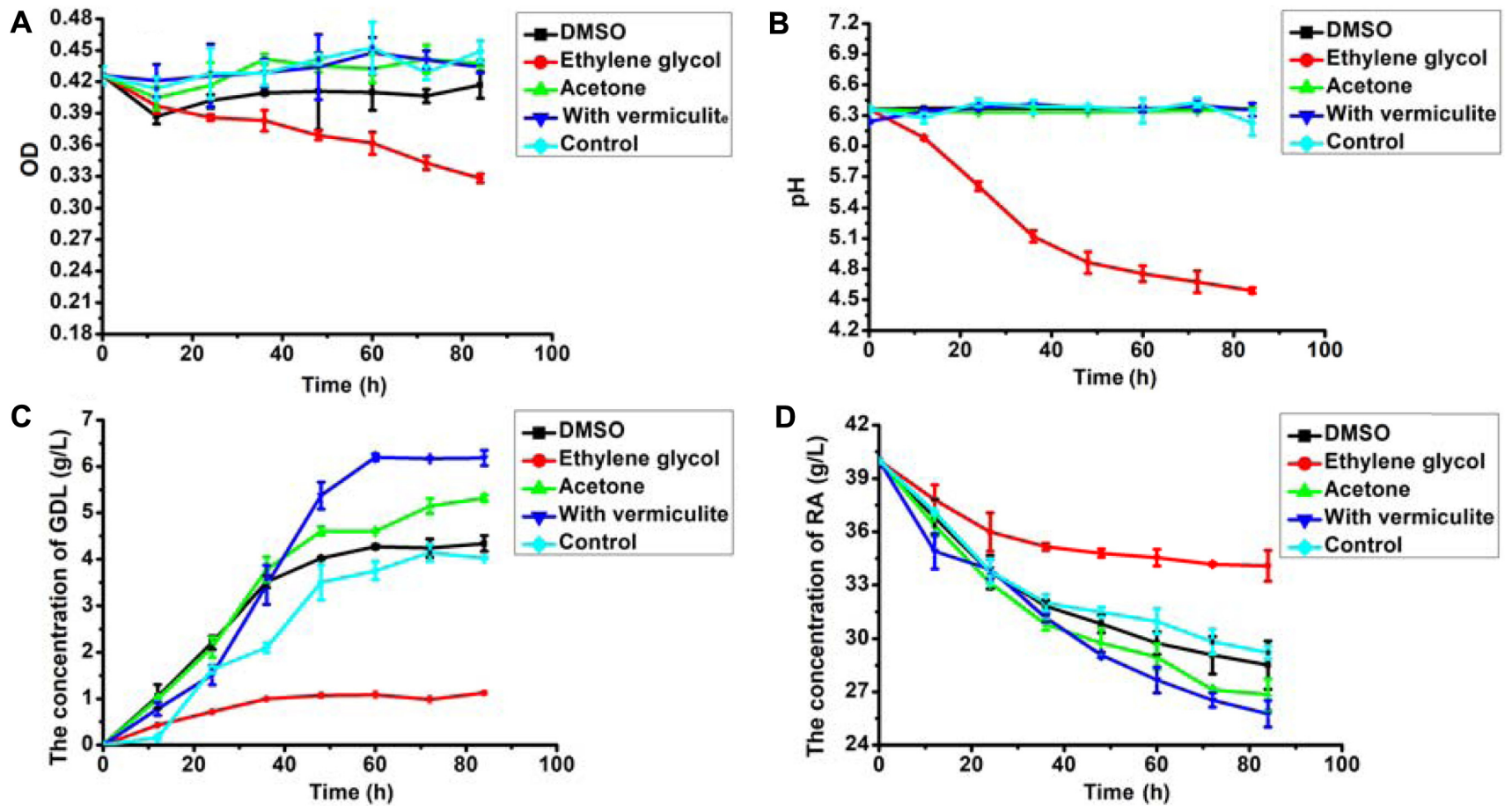

Fig. 1. Changes in yeast cell growth (A) $\mathrm{pH}(\mathbf{B})$ GDL concentration (C) and RA concentration (D) during the cultivation. Error bars show standard errors of means of three replicates.

reference medium were $28.51 \mathrm{~g} / \mathrm{l}$ and $29.21 \mathrm{~g} / \mathrm{l}$, respectively, which were almost the same. The highest conversion rate of RA to GDL was achieved in the medium with E-V, which was $88.3 \%$ at $60 \mathrm{~h}$ (Table 1). For the media with DMSO and acetone and the reference medium, the conversion rates were $72.9 \%, 76.9 \%$, and $78.9 \%$, respectively, at $48 \mathrm{~h}$. The lowest conversion rate was obtained in the medium with ethylene glycol, which was $36.1 \%$. During the biotransformation process, the conversion rates in the cultures with DMSO, acetone, and E-V and the reference culture showed an increasing trend before slightly declining, except for the culture with ethylene glycol, in which the conversion rate consistently declined. Hence, the addition of ethylene glycol could strongly inhibit biotransformation productivity. The addition of E-V had a much less negative effect on the metabolism of Y. lipolytica,

Table 1. The conversion rate of RA to GDL.

\begin{tabular}{lcccccccc}
\hline \multicolumn{1}{c}{ Time (h) } & 0 & 12 & 24 & 36 & 48 & 60 \\
\hline Control (\%) & 0 & $19.6 \pm 2.3$ & $46.0 \pm 3.3$ & $56.0 \pm 0.4$ & $72.9 \pm 5.4$ & $71.9 \pm 1.8$ & $71.4 \pm 1.8$ & $68.3 \pm 2.0$ \\
DMSO (\%) & 0 & $57.9 \pm 4.4$ & $61.9 \pm 5.3$ & $75.3 \pm 0.1$ & $76.9 \pm 3.6$ & $73.1 \pm 3.8$ & $64.9 \pm 3.1$ & $66.5 \pm 5.3$ \\
Ethylene glycol (\%) & 0 & $36.1 \pm 10.5$ & $32.3 \pm 8.4$ & $35.9 \pm 0.2$ & $35.9 \pm 0.7$ & $34.8 \pm 0.02$ & $29.6 \pm 2.3$ & $33.5 \pm 3.4$ \\
Acetone (\%) & 0 & $48.6 \pm 5.8$ & $53.4 \pm 5.2$ & $71.9 \pm 2.7$ & $78.9 \pm 4.8$ & $73.3 \pm 4.5$ & $69.9 \pm 1.5$ & $67.0 \pm 3.8$ \\
With E-V (\%) & 0 & $26.8 \pm 0.3$ & $43.2 \pm 2.2$ & $69.7 \pm 6.7$ & $86.3 \pm 3.5$ & $88.3 \pm 4.3$ & $80.4 \pm 2.2$ & $76.2 \pm 2.0$ \\
\hline
\end{tabular}

Table 2. The production rate of GDL.

\begin{tabular}{lcccccccc}
\hline \multicolumn{1}{c}{ Time $(\mathrm{h})$} & 0 & 12 & 24 & 36 & 48 & 60 & 72 & 84 \\
\hline Control $(\mathrm{g} / \mathrm{l} \cdot \mathrm{h})$ & 0 & $0.013 \pm 0.0035$ & $0.136 \pm 0.0059$ & $0.175 \pm 0.0082$ & $0.292 \pm 0.032$ & $0.313 \pm 0.0165$ & $0.345 \pm 0.0153$ & $0.335 \pm 0.0012$ \\
DMSO $(\mathrm{g} / \mathrm{l} \cdot \mathrm{h})$ & 0 & $0.0871 \pm 0.022$ & $0.184 \pm 0.012$ & $0.293 \pm 0.011$ & $0.335 \pm 0.002$ & $0.356 \pm 0.004$ & $0.353 \pm 0.016$ & $0.362 \pm 0.014$ \\
Ethylene glycol $(\%)$ & 0 & $0.036 \pm 0.004$ & $0.060 \pm 0.001$ & $0.083 \pm 0.003$ & $0.089 \pm 0.006$ & $0.09 \pm 0.008$ & $0.082 \pm 0.008$ & $0.094 \pm 0.004$ \\
Acetone $(\mathrm{g} / \mathrm{l} \cdot \mathrm{h})$ & 0 & $0.083 \pm 0.002$ & $0.175 \pm 0.018$ & $0.315 \pm 0.022$ & $0.384 \pm 0.008$ & $0.384 \pm 0.001$ & $0.429 \pm 0.014$ & $0.443 \pm 0.006$ \\
E-V $(\mathrm{g} / \mathrm{l} \cdot \mathrm{h})$ & 0 & $0.065 \pm 0.012$ & $0.126 \pm 0.018$ & $0.288 \pm 0.035$ & $0.448 \pm 0.024$ & $0.517 \pm 0.006$ & $0.514 \pm 0.001$ & $0.515 \pm 0.014$ \\
\hline
\end{tabular}




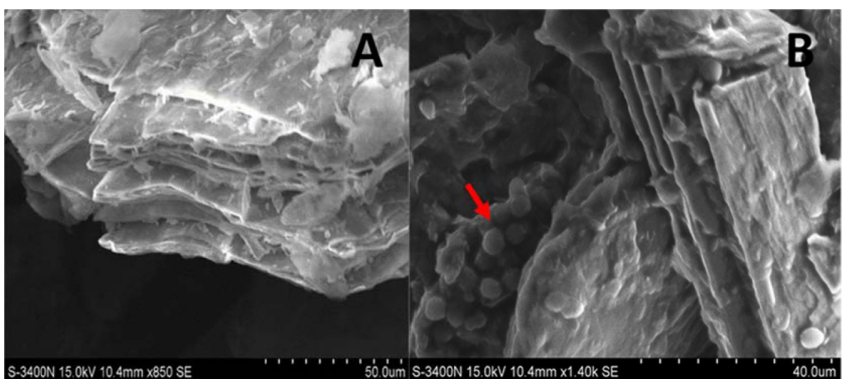

Fig. 2. SEM images of the E-V (A) and E-V-RA (B).

which could improve the utilization of substrate in the fermentation broth.

\section{Characterization of the E-V-RA complexes}

Micro-morphology analysis. Fig. 2 shows SEM images of E-V and E-V coated with the substrate (RA) and $Y$. lipolytica. As shown in Fig. $2 \mathrm{~A}, \mathrm{E}-\mathrm{V}$ has a regular, expandable lamellar structure, which gives it a larger specific surface area. The RA was adsorbed onto the interlayer lamellar structure and the surface of E-V, which increased the contact opportunity between $Y$. lipolytica and RA as they aggregated on the $\mathrm{E}-\mathrm{V}$ and was conducive to a higher output, as indicated by the red point in Fig. 2B.

FTIR spectra analysis. Fig. 3 shows the FTIR spectra of E-V, RA and the E-V-RA complex, which provide structural information. The peaks of the E-V-RA complex (Fig. 3a) showed that the asymmetrical stretching vibrations of Si$\mathrm{O}-\mathrm{Si}$ and $\mathrm{Si}-\mathrm{O}-\mathrm{Al}$ and the bending vibrations of $\mathrm{Si}-\mathrm{OH}$ appeared at 987 and $710 \mathrm{~cm}^{-1}$, respectively, which were identical to the characteristic peaks of E-V (Fig. 3b). These results suggested that the structure of E-V was unaltered. Due to the alkene C-H bending vibration of RA (Fig. 3c), the fingerprint peaks at 1,430 and $1,280 \mathrm{~cm}^{-1}$ were also

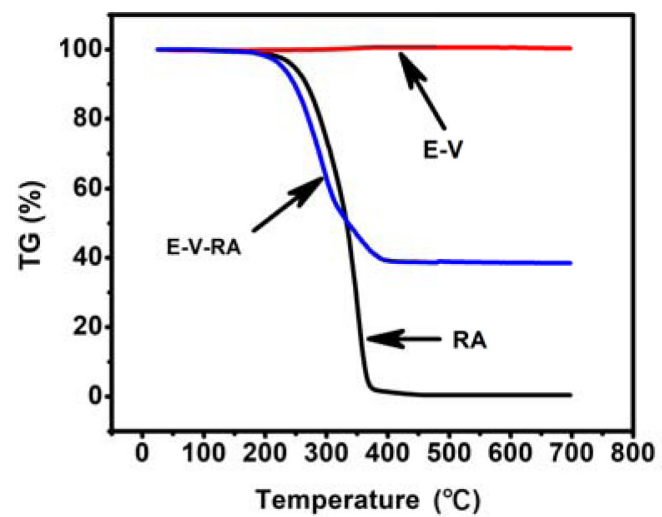

Fig. 4. TG and DTG curves of E-V, E-V-RA and RA.

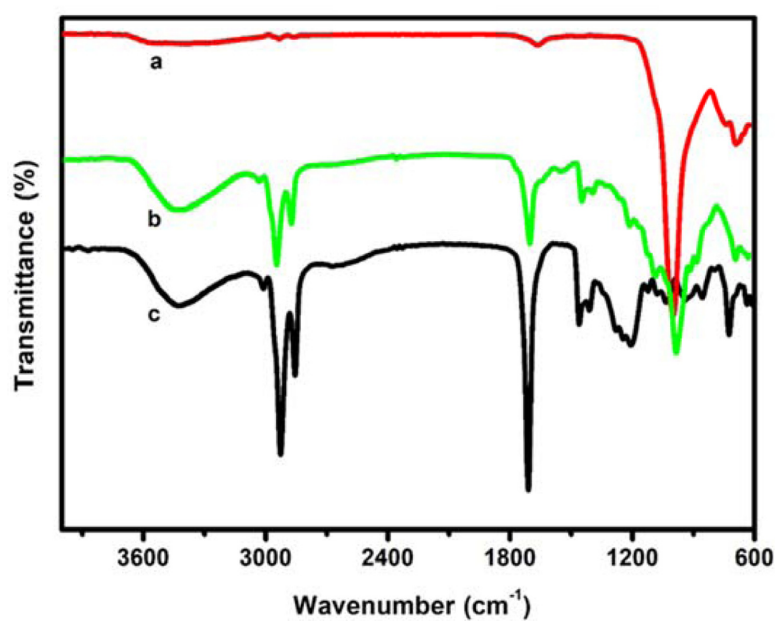

Fig. 3. FTIR of E-V-RA(a), E-V(b) and RA(c).

present in Fig. 3b, suggesting the adsorption of RA onto the $\mathrm{E}-\mathrm{V}$ surface. However, the stretching vibrational peaks of $=\mathrm{C}-\mathrm{H},-\mathrm{C}=\mathrm{O}$ and $-\mathrm{CH}_{2}$ of RA at $3,010,1,710$, and $2,926 \mathrm{~cm}^{-1}$, respectively, in Fig. 3b were weaker than those in Fig. 3c. No new characteristic peaks appeared, which indicated the formation of adsorption complexes between RA and E-V instead of a chemical reaction.

Thermogravimetric (TG) analysis and differential thermogravimetric (DTG) analysis. Thermogravimetric (TG) analysis was carried out to provide supporting evidence for the FTIR results. As shown in Fig. 4, no obvious variation in the curve of $\mathrm{E}-\mathrm{V}$ was observed in the examined temperature range. RA was stable at temperatures below $200^{\circ} \mathrm{C}$. However, when the temperature increased from $200^{\circ} \mathrm{C}$ to $377^{\circ} \mathrm{C}, 96 \%$ weight loss occurred at a DTG temperature of $362^{\circ} \mathrm{C}$. There was also a distinct weight loss for the E-V-RA complex from $250^{\circ} \mathrm{C}$ to $407^{\circ} \mathrm{C}$, which represents the degradation of RA (Fig. 4A) at a DTG

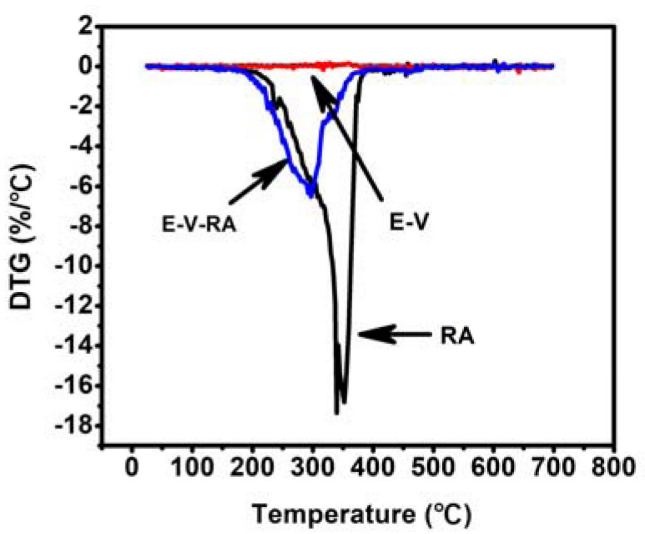


temperature of $297^{\circ} \mathrm{C}$. It could be speculated that the weight loss in the adsorption complex was mostly attributed to the decomposition of RA. In addition, an approximately $62.49 \%$ weight loss was observed for the EV-RA adsorption complex. This result indicates that the composite structure did not influence the thermostability of RA and E-V. Only physical adsorption occurred between RA and E-V, instead of the formation of chemical bonds. The calculated proportions of the weight losses suggested that the adsorption capacity of E-V was $1.5 \mathrm{~g} / \mathrm{g}$.

\section{Discussion}

From the comparative results for the culture with E-V and the three organic solvents above, we conclude that E-V has a better effect on the biotransformation of GDL. In this biotransformation process, E-V acted as a controlled delivery agent to constantly release RA and thus improved its utilization efficiency. The bioconversion rate of RA to GDL was higher in the medium with $\mathrm{E}-\mathrm{V}$, and the highest yield of GDL was $6.2 \mathrm{~g} / \mathrm{l}$, achieved at $60 \mathrm{~h}$, which was 50\% higher than the reference yield. As shown in Figs. 2, 3, and 4 , an adsorption complex between RA and E-V was formed. The mechanism behind this behavior could be interpreted as follows: The layered structure of E-V provides a feasible material for the adsorption of RA due to its accordion-like volume expansion and high specific surface [17-19]. The complexes could be formed by physical adsorption or through covalent or hydrogen bonds between the carboxyl groups in RA and the hydroxyl groups in the inner sphere and outer sphere of EV. It is supposed that the complexes adsorbed on the outer sphere are readily released. However, the complexes bound in the interlayer space might be stronger and more protected against desorption. This adsorption mechanism determines the effective control of the RA release rate and the recycling of E-V.

In addition, $Y$. lipolytica could adhere to the surface and interlayer of $\mathrm{E}-\mathrm{V}$, which was more conducive to GDL biotransformation. The productivity was compared with that of cell immobilization methods reported previously. Many different materials used to produce GDL were compared, and the maximum yield was $1.597 \pm 34 \mathrm{mg} / \mathrm{l}$, obtained from $Y$. lipolytica immobilized on polymethacrylate (DupUM) after $264 \mathrm{~h}$ [20]. The highest yield of GDL was $4.17 \mathrm{~g} / \mathrm{l}$, which was obtained from Y. lipolytica immobilized on a mixture of sodium alginate and attapulgite [21]. However, due to their poor mechanical properties, immobilized carriers are liable to crack. In addition, the mass transfer rate is deeply influenced by the pore structure of the carrier. The productivity of the process is not as high as that in free cells despite the enhanced maximum aroma concentration based on the cell recycling usage. Compared with the cell immobilization method, utilizing an E-V delivery system to produce GDL has much greater advantages.

Regarding the organic solvents, the yields of GDL were all lower than that in the medium with E-V. Among these other media, the highest yield of GDL was obtained in the medium with acetone, which was $5.32 \mathrm{~g} / \mathrm{l}$. This value was $14 \%$ lower than that in the medium with E-V but $28.5 \%$ higher than the control. However, the lowest cell viability, yield of GDL, $\mathrm{pH}$ and consumption of RA were all obtained in the medium with ethylene glycol. Some studies have indicated that low concentrations of organic solvents have a hormesis effect [22], promote the production of some lipases and release secondary metabolites [23, 24]. Moreover, hydrophilic organic solvents can improve the permeability of the cell membrane by destroying the hydrophobicity of the lipids and increasing the flow of lipids or oil-like substrates in the cell membrane [25, 26]. DMSO could promote the production of GDL, but it was toxic towards $Y$. lipolytica. It has been reported that $1 \%$ DMSO can induce apoptosis in human lens cells [27], and DMSO has been proven to provoke a c-myc-dependent decrease in ornithine decarboxylase activity, followed by a depletion of intracellular polyamine levels, associated with programmed cell death and cell growth arrest [28].

The toxicity of organic solvents to cells depends mainly on their concentration in the cell membrane. Furthermore, the addition of organic solvents would cause inconvenience in separation and purification due to the intersolubility of acetone and GDL. Compared with the addition of organic solvents, E-V has no negative effect on cells and forms a stable delivery system. Applying E-V as a delivery system in the biotransformation of GDL to increase the GDL yield is a potential method of improving natural GDL production on an industrial scale.

\section{Acknowledgments}

This work was supported by grants from the Shanghai Sailing Program (Grant No. 17YF1419100), Shanghai Plateau Science Open Fund Program (Grant No. 1021ZK183011004), Shanghai Municipal Education Commission Funded University Experimental Technology Team Construction Project (Grant No. 39110N183001) and Training Plan Of Young Teachers In Universities (Grant No. ZZZZyyx16011). 


\section{Conflict of Interest}

The authors have no financial conflicts of interest to declare.

\section{References}

1. Mosandl A, Gunther C. 1989. Stereoisomeric flavor compounds 20: Structure and properties of $\gamma$-lactone enantiomers. $J$. Agric. Food Chem. 37: 413-418.

2. Longo MA, Sanroman MA. 2006. Production of food aroma compounds: microbial and enzymatic methodologies. Food Technol. Biotechnol. 44: 335-353.

3. Braga A, Belo I. 2015. Production of g-decalactone by Yarrowia lipolytica: insights into experimental conditions and operating mode optimization. J. Chem. Technol. Biotechnol. 90: 559-565.

4. Blin-Perrin C, Molle D, Dufosse L, Le-Quere J L, Viel C, Mauvais $G$, et al. 2000. Metabolism of ricinoleic acid into $\gamma$ decalactone: $\beta$-oxidation and long chain acyl intermediates of ricinoleic acid in the genus Sporidiobolus sp. FEMS Microbiol. Lett. 188: 69-74.

5. Soares GPA, Souza KST, Vilela LF. 2017. $\gamma$-decalactone production by Yarrowia lipolytica and Lindnera saturnus in crude glycerol. Prep. Biochem. Biotechnol. 47: 1-5.

6. Gomes N, Braga A, Teixeira JA, Belo I. 2013. Impact of lipasemediated hydrolysis of castor oil on $\gamma$-decalactone production by Yarrowia lipolytica. J. Am. Oil Chem. Soc. 90: 1131-1137.

7. Moradi H, Asadollahi MA, Nahvi I. 2016. Optimaztion of gamma-decalactone production by yeast Yarrowia lipolytica using the taguchi method. J. Microbiol. Biotechnol. Food Sci. 6: 685-688.

8. Rong S, Wang M, Yang S, Li Q, Guan S, Cai B, et al. 2017. Improvement of $\gamma$-decalactone production by stimulating the import of ricinoleic acid and suppressing the degradation of $\gamma$-decalactone in Saccharomyces cerevisiae. Biocatal. Biotransformation 35: 96-102.

9. Gomes N, Wache Y, Teixeira JA, Belo I. 2011. Oil-in-water emulsions characterization by laser granulometry and impact on gamma-decalaclone production in Yarrowia lipolytica. Biotechnol. Lett. 33: 1601-1606.

10. Aguedo M, Waché Y, Coste F, Husson F, Belin JM. 2004. Impact of surfactants on the biotransformation of methyl ricinoleate into $\gamma$-decalactone by Yarrowia lipolytica. J. Mol. Catal. B-Enzym. 29(s1-6): 31-36.

11. Glover RE, Smith RR, Jones MV, Jackson SK, Rowlands CC. 1999. An EPR investigation of surfactant action on bacterial membranes. FEMS Microbiol. Lett. 177: 57-62.

12. Lin SJ, Lee SL, Chou CC. 1996. Effects of various fatty acid components of castor oil on the growth and production of $\gamma$-decalactone by Sporobolomyces odorus. J. Ferment Bioeng. 82: 42-45.
13. Mouzdahir YE, Elmchaouri A, Mahboub R, Korili SA. 2009. Synthesis of nano-layered vermiculite of low density by thermal treatment. Powder Technol. 189: 2-5.

14. Duman O, Tunç S. 2008. Electrokinetic properties of vermiculite and expanded vermiculite: effects of $\mathrm{pH}$, clay concentration and mono- and multivalent electrolytes. Sep. Sci. Technol. 43: 3755-3776.

15. Duman O, Tunç S, Polat TG. 2015b. Determination of adsorptive properties of expanded vermiculite for the removal of C. I. basic red 9 from aqueous solution: kinetic, isotherm and thermodynamic studies. Appl. Clay Sci. 109110: 22-32.

16. Braga A, Mesquita DP, Amaral AL, Ferreira EC, Belo I. 2015. Aroma production by Yarrowia lipolytica in airlift and stirred tank bioreactors: differences in yeast metabolism and morphology. Biochem. Eng. J. 93: 55-62.

17. Marcos C, Rodriguez I. 2010. Expansion behaviour of commercial vermiculites at $1000^{\circ} \mathrm{C}$. Appl. Clay Sci. 48: 492-498.

18. Marcos C, Arango YC, Rodríguez I. 2009. X-ray diffraction studies of the thermal behaviour of commercial vermiculites. Appl. Clay Sci. 42: 368-378.

19. Xu B, Ma H, Lu Z, Li Z. 2015. Paraffin/expanded vermiculite composite phase change material as aggregate for developing lightweight thermal energy storage cementbased composites. Appl. Energy 160: 358-367.

20. Adelaide B, Isabel B. 2013. Immobilization of Yarrowia lipolytica for aroma production from castor oil. Appl. Biochem. Biotechnol. 169: 2202-2211.

21. Zhao YP, Yan XU, Wang D. 2012. Production capacity of $\gamma$-decalactone increased by using immobilized Yarrowia $s p$. Sci. Technol. Food Indus. 33: 230-233.

22. Shen J, Ditommaso A, Shen M, Lu W , Li Z. 2009. Molecular basis for differential metabolic responses to monosulfuron in three nitrogen-fixing cyanobacteria. Weed Sci. 57: 133-141.

23. Hsieh C, Tsai M J, Hsu T H, Chang D M, Lo C Y. 2005. Medium optimization for polysaccharide production of Cordyceps sinensis. Appl. Biochem. Biotech. 120: 145-157.

24. Niu SC, Duan C, Zhang Q, Duan XH. 2016. Effects of permeabilized treatment on the secretion of glutathione in yeast cells. Sci. Technol. Food Indus. 37: 175-179.

25. Sikkema J, Debont J, Poolman B. 1994. Interactions of cyclic hydrocarbons with biological-membranes. J. Biol. Chem. 269: 8022-8028.

26. Ingram LO, Buttke TM. 1984. Effects of alcohols on microorganisms. Adv. Microb. Physiol. 25: 253.

27. Koike M, Ishino K, Kohno Y, Tachikawa T, Kartasova T, Kuroki T, et al. 1996. DMSO induces apoptosis in SV40transformed human keratinocytes, but not in normal keratinocytes. Cancer Lett. 108: 185-93.

28. Trubiani O, Pieri C, Rapino M, Di PR. 1999.The c-myc gene regulates the polyamine pathway in DMSO-induced apoptosis. Cell Prolif. 32: 119-129. 